\title{
Changes in magnetic resonance imaging scores and ventilation inhomogeneity in children with cystic fibrosis pulmonary exacerbations
}

To the Editor:

Acute pulmonary exacerbations (aPEs) in patients with cystic fibrosis (CF) often result in incomplete recovery of lung function despite treatment, and are associated with lung function decline over time $[1,2]$. Monitoring is therefore important to detect aPEs early and to track treatment responses. Recent studies testing pulmonary function with multiple breath washout $(\mathrm{MBW})$ have shown that the Lung clearance Index (LCI), the main outcome measure of MBW, is a sensitive tool to measure early changes in the $\mathrm{CF}$ lung $[3,4]$. However, the potential role of MBW and LCI in more advanced CF lung disease is currently unclear. In fact, a recent study demonstrated heterogeneous responses in LCI, with a significant proportion of patients with CF showing worsening of LCI with treatment for aPEs [5]. Resolution of mucus plugging, resulting in recruitment of poorly ventilated areas of the lung to MBW, would potentially explain the discordant changes in pulmonary function testing by spirometry and LCI with treatment.

Magnetic resonance imaging (MRI) of the chest is increasingly used to detect both early changes in the CF lung as well as improvement in response to treatment of aPE, including mucus plugging $[4,6]$. To assess whether improvement in mucus plugging can explain the variability in LCI response in patients with CF treated for aPE, we therefore performed parallel measurements of both MRI and LCI in patients before and after treatment of aPE with intravenous antibiotics.

The study was approved by the research ethics board of the Hospital for Sick Children in Toronto, ON, Canada. Participants were recruited between May 2011 and November 2012 from children presenting to the CF outpatient department with clinical signs of a CF aPE requiring hospital admission and treatment with intravenous antibiotics. Following informed written consent from patients and/or caregivers, MRI of the chest was performed (1.5 Tesla scanner; Avanto; Siemens Medical Solutions, Erlangen, Germany). The study protocol included the following sequences: 1) ECG-gated steady state free precession localiser images in the three orthogonal planes; 2) T2-weighted BLADE sequence with periodically rotated overlapping parallel lines with enhanced reconstruction in axial and coronal orientations; and 3) T1-weighted volumetric interpolated breath-hold examination (VIBE) before and after injection of gadopentetate dimeglumine (Magnevist; Bayer, Leverkusen, Germany). Lung morphometry (bronchiectasis, airway thickening, mucus plugging, parenchyma and trapped air) was quantified using the previously published CF-MRI scoring method [7, 8]. CF-MRI is an adapted version of the Brody score for MRI. Each lung lobe was scored independently, and the lingula was evaluated as a sixth lobe. Scores were expressed as a percentage of the maximum score. $\mathrm{MBW}$ to determine LCI was performed using sulfur hexafluoride $\left(\mathrm{SF}_{6}\right)$ and a mass spectrometry-based apparatus (AMIS 2000; Innovision), as previously described [9]. Spirometry measures were analysed as percent predicted. MRI, followed by MBW and spirometry, was performed within $48 \mathrm{~h}$ of admission and after 12 days (range 9-16 days) of intravenous antibiotics for aPEs, respectively.

The study population was summarised using parametric methods for normally distributed data and nonparametric methods for skewed data where necessary. Comparisons before and after treatment were made using paired t-tests. Correlations between MRI and LCI outcomes at pre- and post-treatment time

@ERSpublications

Resolution of mucus plugging may explain worsening LCI during pulmonary exacerbation treatment in children with CF http://ow.ly/LTLm30dp6hS

Cite this article as: Grasemann $\mathrm{H}$, Ciet $\mathrm{P}, \mathrm{Amin} \mathrm{R}$, et al. Changes in magnetic resonance imaging scores and ventilation inhomogeneity in children with cystic fibrosis pulmonary exacerbations. Eur Respir J 2017; 50: 1700244 [https://doi.org/10.1183/13993003.00244-2017]. 


\begin{tabular}{|c|c|c|}
\hline & Before treatment & After treatment \\
\hline Bronchiectasis & $18.8(11.9-25.6)$ & $18.9(13.8-23.9)$ \\
\hline Airway thickening & $15.1(10.0-20.2)$ & $13.5(10.1-16.9)$ \\
\hline Parenchyma & $4.1(2.8-5.4)$ & $4.5(3.1-6.0)$ \\
\hline Mucus plugging & $9.2(6.4-12.1)$ & $5.8(4.0-7.6)^{*}$ \\
\hline Air trapping & $5.3(3.4-7.2)$ & $2.8(1.2-4.3)^{*}$ \\
\hline Total lung score & $52.4(37.6-67.3)$ & $45.4(33.8-57.0)$ \\
\hline
\end{tabular}

Data are presented as mean $\left(95 \% \mathrm{CI} .^{*}: \mathrm{p}<0.05\right.$, comparing scores before and after treatment.

points, as well as correlations between the changes in outcomes, were assessed using Pearson's correlation coefficient.

In total, 13 patients (seven male, six female) underwent MRI before and after antibiotic treatment for aPE. Mean age of the study participants was 13.5 years (range 7.8-17.4 years). Mean \pm SD forced expiratory volume in 1 second (FEV1) was $67 \pm 16 \%$ before treatment, and increased to $74 \pm 14 \%$ after treatment $(\Delta 7 \%$, 95\% CI 2-12\%; $\mathrm{p}=0.01)$. Improvement was also seen for forced vital capacity (FVC) ( $\mathrm{p}=0.04), \mathrm{FEV} 1 / \mathrm{FVC}$ $(\mathrm{p}=0.05)$ and forced expiratory flows at mid lung volumes $(F E F 25-75)(\mathrm{p}=0.05)$. CF-MRI scores before and after treatment are given in table 1.

CF-MRI scores improved significantly for mucus plugging $(\Delta-3.5,95 \%$ CI -5.4 to $-1.5 ; \mathrm{p}=0.002)$ and air trapping $(\Delta-2.5,95 \%$ CI -4.7 to $-0.3 ; \mathrm{p}=0.03)$, whereas no significant changes were seen for bronchiectasis $(\mathrm{p}=0.96)$, airway thickening $(\mathrm{p}=0.27)$, parenchyma $(\mathrm{p}=0.49)$ or total lung score $(\mathrm{p}=0.09)$.

Of the 13 patients who underwent MRI, eight had research-quality MBW measured before and after treatment. Mean \pm SD LCI score was $12.4 \pm 2.3$ before treatment, and did not change with treatment $(\Delta-0.2$, $95 \% \mathrm{CI}-1.1$ to $0.7 ; \mathrm{p}=0.59)$. LCI before treatment was positively correlated with CF-MRI total lung score $(\mathrm{r}=0.64 ; \mathrm{p}=0.06)$, mucus plugging score $(\mathrm{r}=0.72 ; \mathrm{p}=0.03)$ and bronchiectasis score $(\mathrm{r}=0.70 ; \mathrm{p}=0.04)$. However, there was an inverse correlation between changes in LCI and changes in CF-MRI total lung score $(\mathrm{r}=-0.85 ; \mathrm{p}<0.01)$. Significant negative correlations suggested worsening of LCI (increase in LCI) with improvement in mucus plugging $(\mathrm{r}=-0.74 ; \mathrm{p}=0.04)$ and air trapping $(\mathrm{r}=-0.79 ; \mathrm{p}=0.02)$ after therapy (decrease in CF-MRI scores) (figure 1). There was no correlation between changes in MRI scores and changes in spirometry with therapy.

Consistent with previous studies, MRI can be used to quantify lung disease severity in children with $\mathrm{CF}$, and measures of lung function by LCI correlated with CF-MRI scores at the time of aPE. Interestingly, there was considerable individual variability in response to treatment in both CF-MRI scores and LCI. However, in this study, CF-MRI scoring of improved mucus plugging and trapped air following treatment of aPE correlated with worsening LCI. This observation may be best explained by recruitment of poorly ventilated areas of the lung through improvement of mucus plugging with treatment, leading to worsening
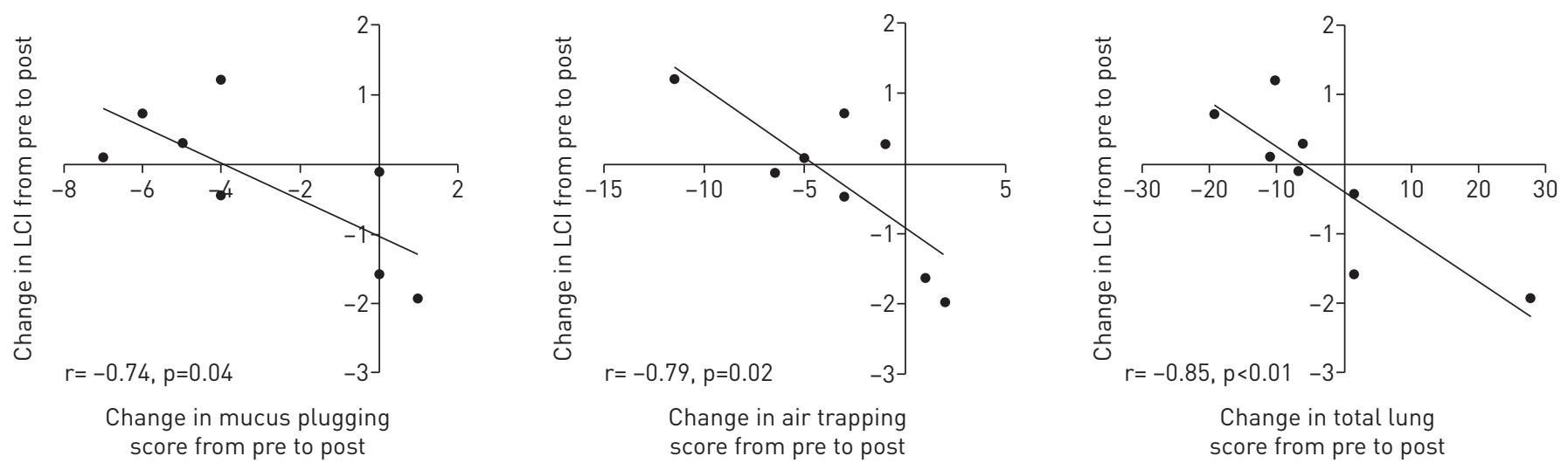

FIGURE 1 Correlations between change in Lung Clearance Index (LCI) and changes in cystic fibrosis magnetic resonance imaging scores from pre- to post-treatment for acute pulmonary exacerbations. Significant negative correlations suggested worsening of LCl (increase in LCI) with improving mucus plugging and air trapping with therapy. 
in LCI. This is consistent with the heterogeneous responses in LCI during treatment of pulmonary exacerbations in older children, which we reported previously [5]. Interestingly, a recent study in children with CF found a more consistent pattern of improvement in LCI with treatment of aPE [4]. Their study included younger children with better pulmonary function and therefore probably less structural lung damage, for whom the net effect of reducing mucus plugging is more likely to improve ventilation homogeneity. While other factors than age and disease severity may contribute to the discrepant results in the two studies, these data would support the concept that LCI as a measure of treatment response may be less informative in individuals with significant airflow obstruction compared with those with early airway disease, as previously suggested [10].

A limitation of our study is the relatively small sample size of patients who had both MRI and LCI measurements performed before and after treatment for aPE. Our findings therefore need to be interpreted with caution and confirmed in a larger population. Further studies are also needed to clarify the role of MBW and chest imaging techniques such as MRI for monitoring treatment responses other than those to antibiotics for aPEs in patients with $\mathrm{CF}$ and moderate to severe lung disease.

Hartmut Grasemann ${ }^{1,2}$, Pierluigi Ciet ${ }^{3,4}$, Reshma Amin ${ }^{1}$, Nancy McDonald $^{1}$, Michelle Klingel ${ }^{2}$, Harm A.W.M. Tiddens ${ }^{3,4}$, Felix Ratjen ${ }^{1,2}$ and Lars Grosse-Wortmann ${ }^{5}$

${ }^{1}$ Division of Respiratory Medicine, Dept of Pediatrics, Hospital for Sick Children, University of Toronto, Toronto, ON, Canada. ${ }^{2}$ Program in Physiology and Experimental Medicine, Research Institute, Hospital for Sick Children, University of Toronto, Toronto, ON, Canada. ${ }^{3}$ Dept of Pediatric Pulmonology, Erasmus MC/Sophia Children's Hospital, Rotterdam, The Netherlands. ${ }^{4}$ Dept of Radiology and Nuclear Medicine, Erasmus MC/Sophia Children's Hospital, Rotterdam, The Netherlands. ${ }^{5}$ Labatt Family Heart Centre, Dept of Pediatrics and Dept of Diagnostic Imaging, Hospital for Sick Children, University of Toronto, Toronto, ON, Canada.

Correspondence: Hartmut Grasemann, Hospital for Sick Children, 555 University Avenue, Toronto, ON, M5G 1X8, Canada. E-mail: hartmut.grasemann@sickkids.ca

Received: Feb 022017 | Accepted after revision: May 292017

Conflict of interest: Disclosures can be found alongside this article at erj.ersjournals.com

\section{References}

1 Stanojevic S, McDonald A, Waters V, et al. Effect of pulmonary exacerbations treated with oral antibiotics on clinical outcomes in cystic fibrosis. Thorax 2017; 72: 327-332.

2 Waters V, Stanojevic S, Ratjen F. Special considerations for the treatment of pulmonary exacerbations in children with cystic fibrosis. Expert Rev Respir Med 2016; 10: 1221-1228.

3 Stanojevic S, Davis SD, Retsch-Bogart G, et al. Progression of lung disease in preschool patients with cystic fibrosis. Am J Respir Crit Care Med 2017; 195: 1216-1225.

4 Stahl M, Wielpütz MO, Graeber SY, et al. Comparison of lung clearance index and magnetic resonance imaging for assessment of lung disease in children with cystic fibrosis. Am J Respir Crit Care Med 2017; 195: 349-359.

5 Sonneveld N, Stanojevic S, Amin R, et al. Lung clearance index in cystic fibrosis subjects treated for pulmonary exacerbations. Eur Respir J 2015; 46: 1055-1064.

6 Wielpütz MO, Puderbach M, Kopp-Schneider A, et al. Magnetic resonance imaging detects changes in structure and perfusion, and response to therapy in early cystic fibrosis lung disease. Am J Respir Crit Care Med 2014; 189: 956-965.

7 Tepper LA, Ciet P, Caudri D, et al. Validating chest MRI to detect and monitor cystic fibrosis lung disease in a pediatric cohort. Pediatr Pulmonol 2016; 51: 34-41.

8 Ciet P, Serra G, Bertolo S, et al. Assessment of CF lung disease using motion corrected PROPELLER MRI: a comparison with CT. Eur Radiol 2016; 26: 780-787.

9 Amin R, Subbarao P, Lou W, et al. The effect of dornase alfa on ventilation inhomogeneity in patients with cystic fibrosis. Eur Respir J 2011; 37: 806-812.

10 Horsley A. Lung clearance index in the assessment of airways disease. Respir Med 2009; 103: 793-799. 